\title{
MODULAÇÃO DA MICROBIOTA INTESTINAL POR PROBIÓTICOS COMO ALTERNATIVA PARA O TRATAMENTO DA DEPRESSÃO: UMA REVISÃO BIBLIOGRÁFICA
}

\section{LORENA CONCEIÇÃO SANTOS, ÁUREA WELTER}

\begin{abstract}
RESUMO - A depressão é considerada uma das doenças que mais geram incapacidade em todo o mundo, com mecanismo fisiopatológico ainda inconclusivo, porém estudos tem evidenciado a sua relação com a microbiota intestinal em estado de disbiose. O presente trabalho de revisão bibliográfica teve como objetivo descrever o mecanismo de comunicação entre o eixo intestino-cérebro, a relação entre a disbiose intestinal e manifestação da depressão bem como a modulação da microbiota intestinal, mediante o uso de probióticos, e implicações na sintomatologia relacionada a depressão. Para tanto, a pesquisa bibliográfica foi elaborada a partir de periódicos científicos nacionais e internacionais, publicados no período de 2002 a 2020 e indexados nas bases de dados disponibilizadas no Portal Periódicos CAPES, Scientific Eletronic Library Online - SciELO e Business Source Complete-EBSCO. A importância dos microrganismos intestinais na regulação do eixo intestino-cérebro e nos distúrbios relacionados a essa via, os quais podem estar associados às alterações cerebrais envolvidas no desenvolvimento da depressão, são bem estabelecidos. Estudos clínicos envolvendo idosos, gestantes e público em geral, os quais receberam formulações probióticas, incluindo diferentes cepas bacterianas evidenciaram que, com a modulação da microbiota intestinal, o estresse relacionado a depressão bem como sintomas depressivos manifestados pelos pacientes foram atenuados, quando em comparação com os pacientes do grupo placebo. Mais investigações são necessárias para corroborar com as evidências aqui apresentadas, com vistas a validar o emprego de probióticos como uma alternativa no tratamento da depressão.
\end{abstract}

PALAVRAS-CHAVE - Depressão; Eixo Intestino-Cérebro; Microbioma.

\section{INTRODUÇÃO}

A depressão é um distúrbio mental caracterizado por humor deprimido, triste, vazio ou irritável, perda de interesse ou prazer em atividades que antes tinha apresso, diminuição da energia, sentimento de culpa, baixa autoestima, sono e apetite alterados, acompanhados das funções cognitivas e somáticas alteradas em decorrência da exposição do indivíduo a vivência de luto, trauma psicológico, condição financeira, não apresso pela a própria aparência física, limitações da vida diária devido a doenças crônicas [1]. É considerada uma das doenças que mais gera incapacidade em todo o mundo e se manifesta em indivíduos, independentemente da idade [2]. Os tratamentos convencionais têm como ó alvo o cérebro, com uso de fármacos que atuam aumentando a disponibilidade de neurotransmissores, no entanto, em 5090\% dos casos, podem reincidir os sintomas depressivos [3][5]. Diante disso, compreende-se a importância de se obter novas formas de tratamento para esta patologia de tamanha complexidade.

Estudos evidenciaram a existência de comunicação entre a microbiota intestinal e o sistema nervoso central (SNC), o que provoca mudanças tanto na função como no comportamento do cérebro [6]. Além, disso, sabe-se que a microbiota intestinal desordenada pode ser um contribuinte no desenvolvimento da depressão [3], [4], como verificado em um estudo envolvendo modelo animal (camundongos), onde se comprovou que houve regulação da ansiedade, humor e cognição, nos animais que foram alimentados com probióticos se comparado aos que possuíam o intestino livre de germes [6].

Sendo assim, o eixo intestino-cérebro está envolvido no surgimento dos transtornos depressivos, apesar das ações dos microrganismos do intestino ainda estarem em processo de estudo [7]. Logo, a modulação da microbiota intestinal mediante o uso de probióticos, ganha notoriedade, podendo ser uma estratégia terapêutica com vistas a redução dos sintomas depressivos [8]. Aliado a esse fato, se confirmado o potencial antidepressivo, o uso de probióticos viria acarretar efeitos positivos na vida dos pacientes que buscam por um tratamento com menos efeitos colaterais, e sem período de 
latência, fatores estes responsáveis pela não adesão ao tratamento com os antidepressivos convencionais [8], [9].

Neste contexto, o presente trabalho tem como objetivo descrever o mecanismo de comunicação entre o eixo intestinocérebro, a relação entre a disbiose intestinal e manifestação da depressão bem como a modulação da microbiota intestinal, mediante o uso de probióticos, e implicações na sintomatologia relacionada a depressão.

\section{METODOLOGIA}

A presente revisão bibliográfica foi elaborada a partir de periódicos científicos nacionais e internacionais, publicados no período de 2002 a 2020 e indexados nas bases de dados disponibilizadas no Portal Periódicos CAPES, Scientific Eletronic Library Online - SciELO e Business Source Complete-EBSCO.

\section{DEPRESSÃO}

A depressão afeta globalmente cerca de 300 milhões de pessoas sem idade específica, em casos extremos resulta em mortes por suicídio, que estimam-se ser de aproximadamente 800 mil pessoas por ano, sendo reconhecida como síndrome clínica a mais de 2 mil anos [1], [2], [10].

De acordo com o Manual Diagnóstico Estatístico de Transtornos Mentais (DSM-5) o termo depressão é usado para se referir aos transtornos depressivos, que envolvem múltiplas categorias diagnósticas, como transtorno disruptivo da regulação do humor, transtorno depressivo maior (TDM), transtorno disfórico pré-menstrual e transtorno depressivo bipolar. Como características principais, cita-se a presença de ao menos cinco dos sintomas anteriormente mencionados, a vulnerabilidade do paciente e o tempo de duração [10].

As causas são multifatoriais podendo ser de origem endógena e exógena. A origem endógena se refere a desordens neurobiológicas, as quais são relacionadas às alterações em neurotransmissores que atuam a nível de $\mathrm{SNC}$, e genéticas, em que há alterações em genomas com chances de herbabilidade (coeficiente de variação genética) entre 37-48\%. Já os fatores exógenos são referidos como fatores psicossociais, os quais são caracterizados como os eventos estressantes da vida, que quando precoce, ainda na juventude, podem ser um grave indutor de depressão, e, os fatores ambientais, como mudanças no perfil da composição da microbiota residente do trato gastrointestinal (TGI), a qual tem papel importante no distúrbio da depressão [4], [11], [12].

Dentre tantas causas, o desequilíbrio de neurotransmissores é considerado inseparável do transtorno da depressão. No que se refere a isto, pode-se mencionar o neurotransmissor 5hidroxitriptamina (5-HT) também denominada de serotonina, que no quadro de depressão se encontra em baixa concentração no SNC, fato este que se acredita ser por alterações no seu metabolismo. Este neurotransmissor é responsável pelo equilíbrio do humor, sensação de bem-estar do ser humano, envolvido na ansiedade e no reconhecimento do medo. Além da 5-HT, a depressão também está associada a neurotransmissores como dopamina e noradrenalina, ambos em desequilíbrio no SNC [4], [11], [12].

Com o avanço da neurociência e bioinformática, foi possível obter evidências de que a fisiopatologia da depressão está ligada com o sistema imunológico, a disfunção do cérebro, o eixo hipotálamo-pituitária-adrenal (HPA), dos quais são vias de acesso entre o cérebro e o intestino, chamado de eixo intestino- cérebro. Logo, está se tornando cada vez mais claro que a depressão é uma doença sistêmica, e não somente neurológica [4].

Apesar dos estudos em humanos serem poucos até o momento, é importante levar em consideração a microbiota intestinal desordenada como sendo influenciadora no aparecimento da depressão [13].

\section{MICROBIOTA INTESTINAL}

A microbiota é definida como um conjunto de microrganismos que possuem um habitat específico [14], 2017) e estão em constante interação com seu hospedeiro [8], sendo que o coletivo de genes de todos os microrganismos é conhecido como microbioma [6]. Constituem a microbiota vírus, fungos, protozoários e em maior quantidade estão as bactérias [15].

A microbiota intestinal é importante para a manutenção das funções fisiológicas do corpo humano por estabelecer uma relação de simbiose, a qual gera benefícios para o hospedeiro [16]. Os filos mais predominantes são Firmicutes e Bacterioidetes e os gêneros mais importantes para a manutenção da saúde do hospedeiro são os Lactobacillus, Clostridium, Enterococcus e Bacteriodes [15].

A alteração da microbiota intestinal é chamada de disbiose, e se caracteriza pela ação de bactérias patogênicas sobre as bactérias comensais, o que gera aumento da permeabilidade das células do intestino e perda de seletividade para absorção de toxinas, bactérias, proteínas ou peptídeos, contribuindo para o aparecimento de doenças, em especial no neurodesenvolvimento, tendo como causas o estresse psicológico e fisiológico, idade, alimentação [5], [17].

\section{EIXO INTESTINO-CÉREBRO}

O intestino pode ser chamado de "segundo cérebro" por este possuir um Sistema Nervoso Entérico (SNE), o qual mantem as funções básicas do intestino, sendo uma delas o peristaltismo. Este sistema atua de forma independente, uma vez que possui um conjunto de neurônios, que em número podem ser comparados aos da medula espinhal e se localizam entre as camadas da musculatura lisa do intestino. A sua constante interação com o cérebro exerce importantes funções no corpo humano [18], [19].

Esta comunicação constante entre o cérebro e o intestino se dá de forma bidirecional, ou seja, o processamento de informações pode iniciar-se no intestino e é encaminhada em direção ao cérebro e vice-versa. Essa dinâmica ocorre por meio de vias neurais como o SNE, nervo vago, nervos simpáticos espinhais, vias humorais como citocinas, hormônios, neuropeptídios bem como o sistema imunológico. Além 
disso, estudos se apoiam na perspectiva de que moléculas liberadas pela microbiota podem exercer efeitos sobre o eixo e influenciar na sua regulação e sinalização, surgindo assim o conceito de microbioma-intestino-cérebro [15], [19], [20].

Visto que existe a possibilidade de obtenção de efeitos benéficos com o bom funcionamento do cérebro e do intestino por meio do equilíbrio da microbiota intestinal, os cientistas buscam compreender o verdadeiro papel do eixo nas vias de comunicação no transtorno depressivo. Há tempos se busca conhecer as causas dos transtornos depressivos estudando somente o cérebro, no entanto, a microbiota em condição de disbiose vem sendo considerada colaboradora no desenvolvimento de doenças psiquiátricas [8], [19], [20].

\section{COMUNICAÇÃO DO EIXO INTESTINO-CÉREBRO}

A microbiota intestinal pode atuar no eixo intestino-cérebro modulando a comunicação bidirecional existente entre os órgãos [21] e, modificações nessas vias de comunicação, são responsáveis por alterações patológicas no indivíduo [15]. A seguir são descritas as vias de comunicação, sendo estas a neural, imunológica e a hormonal.

\section{A. VIA NEURAL}

Esta via é capaz de modular respostas entre o cérebro e a microbiota intestinal e vice-versa [21], sendo mediada pelo nervo vago, Sistema Nervoso Simpática (SNS), Sistema Nervoso Parassimpático (SNP) e SNE.

$\mathrm{O}$ estresse altera a integridade do epitélio intestinal modificando o habitat e a composição da microbiota, bem como a liberação das catecolaminas, as quais alteram a sinalização interbacteriana e os genes de virulência [22].

O SNS reduz a motilidade intestinal por meio da catecolamina noradrenalina, em somatória, o efeito simpático é realizado por modulação inibitória da transmissão colinérgica, assim provoca estímulos no músculo liso causando o retardamento do trânsito intestinal. Em condições de estresse mediados pelo eixo HPA, os ramos simpáticos são ativados e por meio dos nervos esplênicos chegam até a mucosa intestinal onde as células enterocromafins então são ativadas e liberam noradrenalina. Esta atua diretamente no lúmen intestinal onde se encontram os microrganismos que compõe a microbiota, e assim se tem alteração da expressão gênica de bactérias, o que pode resultar em prevalência das bactérias patogênicas sobre as não patogênicas [4], [19].

A comunicação do intestino para o cérebro, de baixo para cima, pode levar informações interoceptivas para este, pois moléculas de sinalização como proteases, histamina, serotonina, citocinas e peptídeos intestinais, são capazes de ativar receptores localizados nas terminações nervosas do nervo vago, os quais são expressos também no núcleo no trato solitário (NTS) e no hipotálamo, levando a crer que os peptídeos intestinais têm síntese estimulada pelas bactérias da flora intestinal e podem ligar-se a esses receptores a nível de SNC [15], [18].

O SNP abrange a maior parte da inervação do intestino, exerce funções inibitórias como o aumento da motilidade, reduz o estímulo motor com a liberação de noradrenalina, neutralizando os efeitos simpáticos. Os nervos parassimpáticos que terminam no nervo vago possuem uma íntima ligação com as bactérias que vivem na luz intestinal, sendo que $80 \%$ desses nervos são aferentes, ou seja, há um volume significativo de informações na comunicação entre o cérebro e o intestino [4]-[6].

O nervo vago possui neurônios aferentes em sua maioria, que levam informações para o pró-encéfalo (região associada a depressão), tálamo, hipocampo, amígdala e córtex préfrontal, e as sinapses vagais ocorrem lateralmente ao núcleo de tronco cerebral [23]. Quando ativado, promove uma resposta anti-inflamatória, defendendo o organismo de sepse causada por microrganismos, ação mediada pelo receptor de acetilcolina, subunidade alfa-7 [15]. Lipopolissacarídeos (LPS) oriundos da microbiota intestinal e, ácidos graxos de cadeia curta (SCFA), provenientes de produtos da fermentação de carboidratos não digeríveis, podem ter acesso a áreas cerebrais que são responsáveis pela cognição e emoções [24].

Já os neurônios eferentes do nervo vago, que são os mais importantes no que se refere a ligação do cérebro-intestino, possuem receptores que são ativados em resposta a nutrientes e substâncias que estão presentes no lúmen intestinal [15], [23]. Em um estudo realizado com o objetivo de determinar o mecanismo de ação do nervo vago, foi examinado o impacto do probiótico Lactobacillus rhamnous no comportamento de receptores centrais do ácido gama-aminobutírico (GABA), principal neurotransmissor inibitório. Concluiu-se que os 36 camundongos objeto de estudo, demonstraram comportamentos de estresse e ansiedade reduzidas por alterações nos receptores GABA A e GABA B. Ao final do experimento estes foram submetidos a vagotomia e continuaram a ser alimentados com o probiótico, porém o efeito ansiolítico não foi demonstrado. Este estudo fornece evidências de que o nervo vago está envolvido nos efeitos neuroquímicos comportamentais presentes na depressão [21].

A falha da sinalização do nervo vago pode ser justificada por danos na microbiota do TGI [6]. Ao estudar camundongos com colite, condição patológica que geralmente é acompanhada de aumento de ansiedade, foi verificado que a vagotomia atenuou este comportamento nos animais, demonstrando que o nervo vago possivelmente esteja relacionado com a inflamação intestinal. No entanto, quando a flora intestinal foi reduzida com o uso de antibióticos, o comportamento de ansiedade ocorreu mesmo sem a ação do nervo vago [23].

Sendo assim, o comportamento de ansiedade verificado nos animais pode ser justificado pelo fato da eliminação de uma via compensar a falta da outra, ou seja, as informações sensoriais que eram transmitidas pelo nervo vago também podem seguir pelas vias imunológicas e/ou hormonal e chegarem ao cérebro.

\section{B. VIA IMUNOLÓGICA}

O sistema imunológico interfere na comunicação bidirecional entre o intestino e o cérebro. As células do sistema imune 
interagem com os microrganismos intestinais por meio da mucosa do TGI e tem comunicação direta com o encéfalo, por meio do nervo vago, ou pelas células imunes presentes na corrente sanguínea [15].

Pacientes com depressão apresentam desregulação do sistema imunológico e inflamação intestinal crônica, o que se deve ao aumento de citocinas pró-inflamatórias, como interleucina-6 (IL-6) e fator de necrose tumoral alfa (TNFalfa) e a diminuição das citocinas anti-inflamatórias, como interleucina 10 (IL-10) e o fator de crescimento tumoral beta (TGF-beta) [4].

As bactérias em grande abundância em pacientes com depressão são gram-negativas, como Oscillibacter, Parabacteroide, Klebsiella, Paraprevotella, Veillonella, Desulfovibrio, Parasutterella e Paraprevotella [?]. Estudos demostraram que as bactérias gram-negativas possuem a capacidade de liberar o LPS, o qual estimula macrófagos a secretarem citocinas pró-inflamatórias (IL-6 e TNF-), além de aumentar a expressão de interferon-gama (INF-) [25], [26]. Logo, a depender do tipo de bactérias presentes no lúmen intestinal, se desenvolve uma resposta inflamatória periférica e as citocinas liberadas pelas células de defesa afetam diretamente a função do SNC. Além disso, em condições de estresse crônico, é alterada a permeabilidade da barreira intestinal, facilitando o aumento e circulação do LPS, componente da parede celular de bactérias gram negativas [6].

O LPS ativa os receptores Toll-like 4 nas micróglias, e estas liberam citocinas inflamatórias, gerando a neuroinflamação [24] o que consequentemente leva ao comprometimento da neuroplasticidade total do SNC, resultando em depressão [4]. O distúrbio da neuroplasticidade pode ser atribuído a redução na produção e biodisponibilidade das monoaminas, a ativação da via da quinurenina e a produção de metabólitos tóxicos como óxido nítrico e espécies reativas de oxigênio (ERO) [27].

Citocinas pró-inflamatórias reduzem a síntese de 5-HT, por ativação da via da quinurenina, a qual também está envolvida na neuroinflamação. O triptofano é convertido em 5-HT em proporcionalidade com a quinurenina. No entanto, em um estado de neuroinflamação, citocinas pró-inflamatórias (IL-6 e TNF-) induzem as enzimas que estimulam a via da quinurenina, a indolamina-2,3-dioxigenase (IDO) e a triptofano-2,3dioxigenase (TDO), assim se tem redução das reservas do substrato para a síntese de 5-HT, principal neurotransmissor envolvido na depressão. Já as citocinas anti-inflamatórias (IL-4 e IL-10) atuam inibindo a via da quinurenina e estimulando a síntese de serotonina [28]. O INF- também induz a IDO, aumentando a síntese da quinurenina a partir do triptofano, e promove a inibição de citocinas anti-inflamatórias como a IL-4 [26].

Sabe-se que em torno de $90 \%$ da 5-HT é produzida no intestino a partir do triptofano [29]. Os microrganismos que compõe a microbiota intestinal, principalmente os Lactobacillus, podem inibir diretamente a IDO por meio da produção de $\mathrm{H} 2 \mathrm{O} 2$, e assim aumentar a expressão de 5-HT circulante. Por outro lado, os metabólitos da via da quinurenina como o ácido quinoliníco, podem alterar o equilíbrio da 5-HT no SNC levando a depressão [26].

Em um estudo envolvendo camundongos alimentados por 120 dias com 108 UFC/dia de Lactobacillus johnsonii, foi constatado que houve redução da atividade metabolizadora da IDO no intestino, tendo-se como consequência a redução da quinurenina circulante e aumento da serotonina periférica. A partir do estudo verificou-se a capacidade do probiótico em produzir $\mathrm{H} 2 \mathrm{O} 2$, o qual é inibidor da enzima IDO, concluindo assim que a redução desta enzima, que é metabolizadora da quinurenina, acarreta em aumento da 5-HT periférica [30].

Quanto aos ácidos graxos de cadeia curta provenientes de bactérias nocivas, estes são capazes de ativar receptores acoplados a proteína $\mathrm{G}$ (Gpr) Gpr-41 e Gpr-43 que são expressos na superfícies das células intestinais e imunológicas provocando uma resposta inflamatória e aumento da permeabilidade intestinal, o que facilita a translocação de metabólitos para a corrente sanguínea, sendo estes capazes de atravessar a barreira hematoencefálica [24].

\section{VIA HORMONAL}

O estresse é considerado fator de risco no desenvolvimento de um quadro depressivo, sendo que $80 \%$ dos episódios de depressão são induzidos por este [28]. Logo, a forma com que o indivíduo lida com ele, determinará a vulnerabilidade para a ocorrência de doenças físicas e psicológicas [31].

A resposta ao estresse está envolvida em dois mecanismos, o SNS e HPA [32]. O SNS é mediado por catecolaminas, que são sintetizadas e estocadas nas células enterocromafins na medula da glândula suprarrenal e encontram-se nas vesículas das terminações nervosas simpáticas. Em condição de estresse, o SNS é ativado, estimulando assim a liberação desses neurotransmissores, o que resulta em taquicardia, aumento da pressão arterial, vasodilatação muscular, reduz a função motora da musculatura do TGI, broncodilatação, aumento da glicogenólise e lipólise [31]. Quando o estresse se dissipa, o SNP libera a acetilcolina, porém em situação de estresse crônico, há o estímulo predominante do SNS, por conseguinte, ocorre a desregulação das catecolaminas que induzem a transcrição de genes responsáveis por desencadear resposta inflamatória [27], a qual foi abordada na via imunológica descrita anteriormente.

As reações de estresse são processadas pelo hipotálamo, induzindo o eixo HPA a partir da liberação do hormônio liberador de corticotrofina $(\mathrm{CRH})$, o qual estimula a adenohipófise a secretar o hormônio adrenocorticotrófico (ACTH). O ACTH, por meio da corrente sanguínea, chega ao córtex da suprarrenal onde estimula a liberação do cortisol, o qual atua na regulação de efeitos corporais em resposta ao estresse, como a decomposição do glicogênio em glicose para repor as necessidades de energia [31]. O aumento da concentração de cortisol circulante inibe a liberação do CRH pelo hipotálamo por feedback negativo [4].

O cortisol é capaz de aumentar a permeabilidade intestinal, por causar a expansão dos complexos juncionais, favorecendo a passagem de citocinas inflamatórias e bactérias 
patogênicas, além disso, pode influenciar na composição, fisiologia e habitat da microbiota intestinal por modificar a expressão dos genes bacterianos [15]. Um estudo propôs uma ligação entre a microbiota intestinal e o eixo HPA, uma vez que em camundongos livres de germes foi verificada uma resposta exagerada ao estresse quando o cortisol estava aumentado, porém com a recolonização da microbiota intestinal o estresse foi reduzido [33]. Ressalta-se aqui que no intestino, as bactérias comensais garantem a integridade das células epiteliais, entre elas os complexos juncionais, que conferem permeabilidade seletiva, ou seja, restringe a passagem de compostos nocivos para o interior do intestino [15].

Alguns pacientes deprimidos têm aumento crônico de cortisol e ACTH, isso se deve a disfunção do feedback negativo do eixo, provocando até hipercortisolemia [4]. O desequilíbrio na composição da microbiota intestinal pode levar a hiperativação do eixo HPA, fato que pode ser sustentado pelo estudo em que foi realizada a administração oral de bactérias patogênicas e verificou-se que estes microrganismos podem atuar na resposta ao estresse pelas vias vagais, evidenciando que a microbiota pode ativar os circuitos de estresse [34]. Além disso, estudos indicam que as citocinas próinflamatórias levam a resistência do receptor glicocorticóide, amplificando a resposta inflamatória e produção excessiva de CRH, e, consequentemente, de cortisol [35].

Além do cortisol, a via hormonal envolve o sistema enteroendócrino composto por células entero-enroendócrinas (EECs) que produzem hormônios como colescistoquinina (CCK) e grelina, os quais atuam regulando o apetite, a síntese de 5-HT e peptídeos intestinais. As EECs podem sofrer interferência no seu funcionamento pela microbiota intestinal, como constatado em um estudo onde camundongos livres de germes que apresentaram alterações na concentração de EECs no íleo e no cólon e consequentemente, houve redução de peptídeos intestinais como CCK, favorecendo a hipótese de que a flora intestinal pode influenciar a síntese de peptídeos [15], [36].

Sendo assim, a desregulação do eixo HPA leva a alterações na motilidade e permeabilidade intestinal bem como na função imunológica desta [6], [8], e portanto, está associada aos episódios de depressão [34].

\section{PROBIÓTICOS}

Os probióticos são microrganismos vivos, que, quando consumidos em quantidades ideais, atuam em benefício da saúde de seu hospedeiro. Nos últimos anos, estes têm sido objeto de estudos entre os cientistas da área médica, industrial e farmacêutica [7], [8], [37].

A incorporação dos probióticos na dieta seja gradual, de maneira a atingir os níveis recomendados em um período de 2-3 semanas, com dose máxima diária de 109-1010 UFC/ml. O consumo de doses superiores pode resultar em flatulências e espasmos intestinais que podem ser resolvidos com o tempo de tratamento, isso se deve pela morte das bactérias patogênicas no intestino liberarem produtos celulares tóxicos [38].
Os probióticos devem ser de origem humana e estáveis ao ácido estomacal e aos sais biliares. Quanto ao mecanismo de ação, este é variado e inclui a adesão a parede do lúmen intestinal, onde irão competir com os microrganismos patogênicos pelos nutrientes impedindo assim a sua proliferação destes; auxiliam no crescimento da mucosa no intuito de manter a função da integridade da barreira epitelial; atuam na modulação da resposta imunológica inata e adaptativa bem como controlando a taxa de proliferação e apoptose das células de defesa [7], [39], [40]. Sendo assim, o uso de probióticos na modulação da microbiota intestinal torna-se uma estratégia interessante no que diz respeito a manutenção e restauração da saúde do indivíduo [3].

A disbiose pode favorecer o aparecimento de doenças, incluindo a depressão, já que mecanismos neurais exercidos pelas bactérias possivelmente são capazes de interferir na ação de neurônios. Logo, a suplementação com probióticos pode ser uma alternativa na modulação da microbiota desordenada, com o intuito de melhorar os sintomas da depressão, principalmente com o uso das bactérias dos gêneros Bifidobacterium e Lactobacillus. Embora o uso terapêutico dos probióticos ter sido estudado para várias doenças, no tratamento da depressão ainda são poucos até o momento [20], [40]-[42].

\section{DISBIOSE INTESTINAL E OS SINTOMAS DEPRESSIVOS}

A microbiota intestinal tem um papel importante na saúde do hospedeiro, como na proteção contra microrganismos patogênicos, no fornecimento de nutrientes essenciais, no metabolismo de fármacos e na absorção e armazenamento de ácidos graxos. O desequilíbrio na microbiota intestinal, é denominado de disbiose [4], [43], [44].

A função cerebral, bem como na regulação do eixo cérebro-intestino, segundo estudos, sofre influências de acordo com estado da microbiota intestinal, ou seja, a sua composição interfere no neurodesenvolvimento, sendo que uma das principais atividades das bactérias comensais é de garantir a integridade da barreira intestinal [4], [45], [46].

Estudos sugerem que o desequilíbrio na microbiota intestinal tem impacto plausível na depressão. Pacientes com depressão apresentam uma redução na composição da microbiota intestinal se comparado aos indivíduos saudáveis [15]. Como consequência da disbiose, há um aumento da permeabilidade intestinal que favorece o extravasamento de substâncias, neste trabalho já mencionadas, como sendo contribuintes para o surgimento da depressão devido a neuroinflamação [24], [26].

Fatores que possivelmente contribuem para a alteração da microbiota intestinal são os antibióticos, fármacos de papel extremamente importante na terapia contra infecções bacterianas, que atuam na redução da concentração das bactérias patogênicas, porém, também das bactérias comensais, levando a disbiose e aumentando a incidência de depressão, de acordo com a vulnerabilidade do indivíduo [4]. Outro fator é a alimentação, que quando rica em lipídeos, esta 
favorece um aumento de bactérias gram-negativas, elevando a concentração de LPS e estimulação do sistema imunológico [26], como acima abordado.

Estudos pré-clínicos indicam a possível relação entre a depressão e a flora intestinal, uma vez que o estresse crônico em modelos animais, levou estes a apresentarem anedonia e modificações na microbiota intestinal, com aumento no ceco de Clostridium sp [47], a qual é uma bactéria gram-negativa. Já em um estudo realizado em primatas, se verificou que o estresse no início da vida tem impacto negativo sobre a flora intestinal, uma vez que nos animais separados da mãe, por um período de 3 dias, se constatou na análise de fezes que houve redução de Lactobacillus, a qual é uma bactéria grampositiva [15].

Bactérias gram-positivas, como os filos Bacteriodetes e Firmicutes, se encontram reduzidos em pacientes com depressão [46]. Ainda, em relação as bactérias gram-positivas, um estudo demonstrou que a reposição da flora intestinal em camundongos com Lactobacillus farciminis reduziu a permeabilidade da parede intestinal, a qual é resultante da hiperatividade do eixo HPA [43].

Dessa forma, é evidente o papel da disbiose em alterações intestinais capazes de modificar as vias de comunicação no eixo intestino-cérebro.

\section{PROBIÓTICOS NO TRATAMENTO DA DEPRESSÃO}

A seguir, são abordados estudos clínicos acerca do uso de probióticos e a resposta terapêutica quanto aos sintomas depressivos.

Para avaliar os efeitos dos probióticos na depressão, foi conduzido um estudo clínico duplo-cego, controlado, randomizado e paralelo que teve duração de 30 dias. Foram incluídos 55 voluntários sadios que não faziam uso de medicamentos, os quais foram divididos em dois grupos: o que recebeu placebo e outro a formulação probiótica (FP) contendo Lactobacillus hervecticus e Bifidobacterium longum na dose $1,5 \mathrm{~g} /$ dia com 3 x 109 UFC/sachê. Ao final do estudo, concluíram que os pacientes que receberam FP tiveram diminuição nos níveis de cortisol em comparação com o grupo placebo e, demostraram redução dos níveis de estresse, sugerindo que FP possui propriedades ansiolíticas e antidepressivas [47]. Pacientes com depressão têm níveis de cortisol aumentados em função da super estimulação do eixo HPA. Como os probióticos diminuem a permeabilidade intestinal, as bactérias gram-negativas produtoras de LPSs não alcançam o SNC, prevenindo assim a estimulação do eixo HPA [34].

Com o intuito de avaliar os benefícios da suplementação com probióticos no transtorno TDM, foi conduzido um ensaio randomizado, duplo-cego e controlado, envolvendo 40 voluntários com TDM, com idades entre 20-55 anos. Do total de pacientes, 20 receberam diariamente por um período de 8 semanas, por via oral, uma FP que continha as cepas Lactobacillus aciddophilus, Lactobacillus casei e Bifidobacterium bifidum, na dose de 2 x $109 \mathrm{UCF} / \mathrm{g}$ e pacientes 20 receberam placebo. Foi constatado que nos pacientes que fizeram uso de FP, houve melhora dos sintomas depressivos, redução do estresse oxidativo e de biomarcadores de inflamação em relação ao grupo placebo [48].

Numa perspectiva de avaliar os efeitos dos probióticos na profilaxia da depressão, realizaram um ensaio randomizado, triplo-cego, controlado por placebo, envolvendo 40 voluntários saudáveis sem uso de nenhum medicamento prescrito. Estes foram divididos em dois grupos: o placebo e o grupo que recebeu diariamente por um período de 4 semana a FP na dose de 2,5 x 109 UFC/g contendo Lactobacillus brevis, Lactobacillus salivarius, Lactobacillus lactis, Bifidobacterium lactis e Bifidobacterium bi-W23. A partir dos resultados do estudo, constataram que a FP provocou considerável redução de pensamentos negativos e repetitivos bem como agressivos associados a tristeza, sugerindo assim que a suplementação com probióticos tem potencial como ação preventiva na depressão [49].

Em uma população constituída por 423 mulheres grávidas (entre $14^{\mathrm{a}}$ e $16^{\mathrm{a}}$ semanas) saudáveis e não faziam uso de fármacos, foi avaliado o efeito de um probiótico. Este estudo foi um ensaio randomizado, duplo-cego e controlado por placebo. Do total de participantes, 380 finalizaram o estudo, sendo que destas 193 receberam o probiótico (Lactobacillus rhamnosus na dose de $6 \times 109 \mathrm{UCF} / \mathrm{g}$ ) diariamente durante o período de gravidez e até 6 meses após o parto e 187 receberam placebo durante esse mesmo prazo de tempo. Foi verificado que durante o período da gravidez e no pósparto, as mulheres que utilizaram o probiótico apresentaram níveis de depressão e ansiedade menores em comparação ao grupo placebo, evidenciando a possibilidade de que a suplementação com probiótico pode ser uma alternativa para a redução dos sintomas depressivos no pós-parto [50].

Objetivando analisar a ação de um probiótico na resposta ao estresse quando da exposição a um teste pressórico de frio, nos padrões de cognição e de atividade cerebral foi conduzido um estudo em humanos do sexo masculino, saudáveis que não utilizavam medicamentos que interferissem no resultado da pesquisa. Participaram 22 voluntários com idades entre 18 e 40 anos sendo que estes receberam placebo por 4 semanas e após esse período, foi administrado por mais 4 semanas, o probiótico Bifidobacterium logum, na dose de 1 x 109 UFC/sachê, sendo este dissolvido no leite. A partir dos resultados do estudo, foi possível concluir que, quando estes pacientes foram submetidos a condição de estresse, ao final do período de uso do placebo, houve um aumento de cortisol e da ansiedade. No entanto, ao serem submetidos a condição de estresse, ao final do período de uso do probiótico, os mesmos parâmetros foram atenuados [51].

\section{CONSIDERAÇÕES FINAIS}

Os microrganismos intestinais interagem com seu hospedeiro com o intuito de manter a homeostasia. A interação com o SNC acontece de forma bidirecional, por meio de vias neurais, hormonais e imunológicas, sendo que, distúrbios na ligação destas vias, podem estar associados às alterações cerebrais que possivelmente induzem ao desenvolvimento da 


\section{depressão.}

O ponto chave deste processo provavelmente é o aumento da permeabilidade intestinal, que pode ser provocado tanto pelo aumento das bactérias patogênicas como pelo estresse crônico. Logo, como consequência, há o extravasamento de bactérias que produzem LPSs, o qual estimula o sistema imunológico a liberar citocinas pró-inflamatórias (TNF- e INF-), provocando inflamação periférica e a nível de SNC. Em condições de neuroinflamação, modifica-se a neuroplasticidade induzindo a desregulação do eixo HPA, redução de neurotransmissores, bem como alterações nas funções do nervo vago, tendo impacto no comportamento do indivíduo.

Os resultados dos estudos aqui abordados demonstraram que a microbiota em estado de disbiose favorece o surgimento da depressão e evidências científicas denotam que os probióticos têm a capacidade de modular a microbiota intestinal, e, provavelmente, por promoverem uma redução da permeabilidade intestinal, estes atenuam o estresse relacionado a depressão bem como sintomas depressivos manifestados pelos pacientes.

No entanto, são necessárias mais evidências, a partir de estudos clínicos do efeito antidepressivo oriundo da modulação da microbiota intestinal, após a administração de probióticos, para assim ampliar o arsenal de fármacos disponíveis no tratamento da depressão.

\section{Referências}

[1] APA - Manual diagnóstico e estatístico de transtornos mentais (DSM-5). American Psychiatric Association. v. 5 ed. Porto Alegre: Artmed; 2014.

[2] OPAS/OMS. Folha informativa: Depressão. 2018.

[3] ALMADA, C. N. de et al. Characterization of the intestinal microbiota and its interaction with probiotics and health impacts. Applied Microbiology and Biotechnology, 99(10):4175-4199, 2015.

[4] LIANG, S. et al. Recognizing depression from the microbiota-gut-brain axis. International Journal of Molecular Sciences, 19(6):1592, 2018.

[5] ZORZO, R. A. Impacto do microbioma intestinal no Eixo CérebroIntestino. International Journal of Nutrology, 10(1):298-305, 2017.

[6] CRYAN, J. F.; DINAN, T. G. Mind-altering microorganisms: the impact of the gut microbiota on brain and behaviour. Nature Reviews Neuroscience, 13(10):701-712, 2012.

[7] TAYLOR, A. M.; HOLSCHER, H. D. A review of dietary and microbial connections to depression, anxiety, and stress. Nutritional Neuroscience, 23(3):237-250, 2018

[8] SILVESTRE, C. M. R. F. O diálogo entre o cérebro e o intestino: qual o papel dos probióticos? 2015. 51 f. Tese- Faculdade de Medicina da Universidade de Lisboa, Lisboa.

[9] WALLACE, C. J. K.; MILEV, R. The effects of probiotics on depressive symptoms in humans: a systematic review. Annals of General Psychiatry, 16(1):1-10, 2017.

[10] BECK, A.; ALFORD, B. A. Depressão: causas e tratamento. 2. ed. São Paulo: Artmed, 2011. 334 p.

[11] GALHARDO, V. A. C.; MARIOSA, M. A. S.; TAKATA, J. P. I. Depressão e perfis sociodemográfico e clínico de idosos institucionalizados sem déficit cognitivo. Revista de Médica de Minas Gerais. 20(1):16-21, 2010.

[12] ZALAR, B.; HASLBERGER, A.; PETERLIN, B. The of microbiota in depression- A brief review. Psychiatria Danubina, 30(2):136-141, 2018.

[13] MARESE, A. C. M. et al. Principais mecanismos que correlacionam a microbiota intestinal com a patogênese da depressão. FAG Journal of Health, 1(3):232-239, 2019.

[14] LIMA-OJEDA, J. M.; RUPPRECHT, R.; BAGHAI, T. C. I Am I and My Bacterial Circumstances: linking gut microbiome, neurodevelopment, and depression. Frontiers in Psychiatry, 8:1-13, 2017.

[15] LACH, G. et al. Envolvimento da flora intestinal na modulação de doenças psiquiátricas. Vittalle - Revista de Ciências da Saúde, 29(1):64-82, 2017.
[16] LESSA, S. S. C.; DELANI, T.; FERREIRA, A. A. Probióticos e prebióticos: ações fisiológicas no sistema digestivo. Brazilian Journal of Surgery And Clinical Research - Bjscr. Maringá, 85-91, 2015.

[17] SARAIVA, F. R. de S.; CARVALHO, L. M. F. de; LANDIM, L. A. dos S R. Depressão e disbiose. Nutrição Brasil, 18(3):175-181, 2020.

[18] MAYER, E. A. Gut feelings: the emerging biology of gut: brain communication. Nature Reviews Neuroscience, 12(8):453-466, 2011.

[19] ESTEVE, I. B. The microbiota-gut-brain axis and its association with depression. 35 f. TCC (Graduação) - Curso de Farmácia e Ciências da Alimentação, Universidade de Barcelona, Barcelona, 2019.

[20] WESTFALL, S. et al. Microbiome, probiotics and neurodegenerative diseases: deciphering the gut brain axis. Cellular and Molecular Life Sciences, 74(20):3769-3787, 2017.

[21] DINAN, T. G. et al. Collective unconscious: how gut microbes shape human behavior. Journal Of Psychiatric Research, 63:1-9, 2015.

[22] BERCIK, P.; COLLINS, S. M.; VERDU, E. F. Microbes and the gut-brain axis. Neurogastroenterology Motility, 24(5):405-413, 2012.

[23] KLARER, M. et al. Gut vagal afferents differentially modulate innate anxiety and learned fear. Journal of Neuroscience, 34(21):7067-7076, 2014.

[24] YARANDI, S. S. et al. Modulatory effects of gut microbiota on the central nervous system: how gut could play a role in neuropsychiatric health and diseases. Journal of Neurogastroenterology and Motility, 22(2):201-212, 2016.

[25] BARANDOUZI, Z. A. et al. Altered composition of gut microbiota in depression: a systematic review. Frontiers in Psychiatry, 11:1-10, 2020.

[26] COBB, C. A Link between gut Mmcrobes depression: microbial activation of the human kynurenine pathway. 2017. $46 \mathrm{f}$. Tese. Curso de The Degree Of Bachelor Of Arts, Claremont Mckenna, Claremont, 2017. Cap. 1.

[27] KIM, Y.; WON, E. The influence of stress on neuroinflammation and alterations in brain structure and function in major depressive disorder Behavioural Brain Research, 329:6-11, 2017.

[28] SANTOS, P. F. da C. R. dos. Neuroinflamação e a Depressão: novas abordagens terapêuticas no horizonte? 33 f. Tese - Curso de Medicina, Faculdade de Medicina da Universidade de Lisboa, Lisboa, 2019.

[29] VEDOVATO, K. et al. O eixo intestino-cérebro e o papel da serotonina. Arquivos de Ciências da Saúde da Unipar, 18(1):33-42, 2015.

[30] VALLADARES, R. et al. Lactobacillus johnsonii inhibits indoleamine 2,3dioxygenase and alters tryptophan metabolite levels in BioBreeding rats. The Faseb Journal, 27(4):1711-1720, 2013.

[31] NODARI, N. L. et al. Estresse, conceitos, manifestações e avaliação em saúde: revisão de literatura. Saúde e Desenvolvimento Humano, Manaus, 2(1):61-74, 2014.

[32] SLAVICH, G. M.; MONROE, S. M.; GOTLIB, I. H. Early parenta loss and depression history: associations with recent life stress in major depressive disorder. Journal of Psychiatric Research, 45(9):1146-1152, 2011.

[33] SUDO, N. et al. Postnatal microbial colonization programs the hypothalamic-pituitary-adrenal system for stress response in mice. The Journal of Physiology, 558(1):263-275, 2004.

[34] BASTIAANSSEN, T. F. S.et al. Gutted! Unraveling the role of the microbiome in major depressive disorder. Harvard Review of Psychiatry, 28(1) 26-39, 2020.

[35] DANTZER, R. et al. From inflammation to sickness and depression: when the immune system subjugates the brain. Nature Reviews Neuroscience, 9(1):46-56, 2008.

[36] DUCA, F. A. et al. Increased Oral Detection, but Decreased Intestina Signaling for Fats in Mice Lacking Gut Microbiota.Plos One, 7(6):1-11, 2012.

[37] SHARIFI, M. et al. Kefir: a powerfull probiotics with anticancer properties. Medical Oncology. 34(11):1-17, 2017.

[38] RAIZEL, R. et al. Efeitos do consumo de probióticos, prebióticos e simbióticos para o organismo humano. Revista Ciência Saúde, 4(2):6674, 2011.

[39] OLIVEIRA, M. N. de et al. Efeitos do consumo de probióticos, prebióticos e simbióticos para o organismo humano. Brazilian Journal of Pharmaceutical Sciences, 38(1):1-21, 2002.

[40] MEDEIROS, A. C.; MAYNARD, D. da C. A influência do microbioma intestinal no desenvolvimento de processos depressivos e o uso de probióticos como tratamento, 2019. 29 f. TCC - Centro Universitário de Brasília - Uniceub, Brasília, 2019.

[41] WANG, Y.; KASPER, L. H. The role of microbiome in central nervous system disorders. Brain, Behavior and Immunity, 38:1-12, 2014. 
[42] VALDES, A. M et al. Role of the gut microbiota in nutrition and health Bmj, p. 36-44, 2018.

[43] FOSTER, J A.; NEUFELD, K. M. Gut-brain axis: how the microbiome influences anxiety and depression. Trends in Neurosciences, 36(5):305312, 2013.

[44] MACEDO, D. et al. Antidepressants, antimicrobials or both? Gut microbiota dysbiosis in depression and possible implications of the antimicrobial effects of antidepressant drugs for antidepressant effectiveness. Journal of Affective Disorders, 208:22-32, 2017.

[45] SAVIGNAC, H. M. et al. The effects of repeated social interaction stress on behavioural and physiological parameters in a stress-sensitive mouse strain. Behavioural Brain Research, 216(2):576-584, 2011.

[46] NASERIBAFROUEI, A. et al. Correlation between the human fecal microbiota and depression. Neurogastroenterology Motility, 26(8):11551162, 2014.

[47] MESSAOUDI, M. et al. Assessment of psychotropic-like properties of a probiotic formulation (Lactobacillus helveticus R0052 and Bifidobacterium longum R0175) in rats and human subjects. British Journal of Nutrition, 105(5):755-764, 2010.

[48] AKKASHEH, $\mathrm{G}$ et al. Clinical and metabolic response to probiotic administration in patients with major depressive disorder: a randomized, double-blind, placebo-controlled trial. Nutrition, 32(3):315-320, 2016.

[49] STEENBERGEN, L. et al. A randomized controlled trial to test the effect of multispecies probiotics on cognitive reactivity to sad mood. Brain, Behavior and Immunity, 48:258-264, 2015.

[50] SLYKERMAN, R.F. et al. Effect of Lactobacillus rhamnosus HN001 in pregnancy on postpartum symptoms of depression and anxiety: a randomised double-blind placebo-controlled trial. Ebiomedicine, 24:159-165, 2017.

[51] ALLEN, A P et al. Bifidobacterium longum 1714 as a translational psychobiotic: modulation of stress, electrophysiology and neurocognition in healthy volunteers. Translational Psychiatry, 6(11):1-7, 2016.

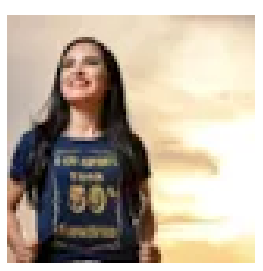

\section{LORENA CONCEIÇÃO SANTOS}

Centro Universitário Luterano de PalmasCEULP/ULBRA, Aureny I, Porto Seguro, NW10A LT1, 77060- 112, Palmas/TO.

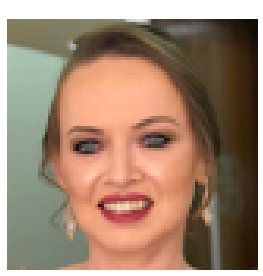

\section{ÁUREA WELTER}

Universidade Federal do Tocantins (UFT) e Centro Universitário Luterano de Palmas CEULP/ULBRA, Universidade Federal do Tocantins (UFT), BALA II, Avenida NS 15, Quadra 109 Norte, Plano Diretor Norte, Campus de Palmas. 